\title{
AN APPROXIMATION TO THE LEAST ROOT OF A CUBIC EQUATION WITH APPLICATION TO THE DETERMINATION OF UNITS IN PURE CUBIC FIELDS
}

BY T. A. PIERCE

1. Approximation to the Least Root of a Cubic. Bernoulli's method of approximating the largest root of an equation

$$
x^{3}=a x^{2}+b x+c,
$$

with real coefficients, is to use (1) as a scale of relation for the recursion formula $A_{n}=a A_{n-1}+b A_{n-2}+c A_{n-3}$. Successive $A$ 's are calculated starting from any initial values. Then $A_{n+1} / A_{n}$ for increasing values of $n$ approximates that root of (1) which has the greatest absolute value if that root is real. The method here given for approximating the least root of (1) is similar to Bernoulli's. We use three recursion formulas

$$
\left\{\begin{array}{l}
A_{n}=a A_{n-1}+b A_{n-2}+c A_{n-3}, \\
B_{n}=a B_{n-1}+b B_{n-2}+c B_{n-3}, \\
C_{n}=a C_{n-1}+b C_{n-2}+c C_{n-3},
\end{array}\right.
$$

and calculate the successive $A$ 's, $B$ 's, and $C$ 's starting from the three sets of initial values

$$
\left\{\begin{array}{l}
\left(A_{-2}, A_{-1}, A_{0}\right)=(0,0,1), \\
\left(B_{-2}, B_{-1}, B_{0}\right)=(0,1,0), \\
\left(C_{-2}, C_{-1}, C_{0}\right)=(1,0,0) .
\end{array}\right.
$$

Then the quotient

$$
-\frac{\left|\begin{array}{ll}
A_{n} & C_{n} \\
A_{n+1} & C_{n+1}
\end{array}\right|}{\left|\begin{array}{ll}
A_{n} & B_{n} \\
A_{n+1} & B_{n+1}
\end{array}\right|}
$$


for increasing values of $n$, approximates the root of (1) of least absolute value if that root is real.

The recursion formulas (2) are linear difference equations and have the solutions

$$
\left\{\begin{array}{l}
A_{n}=\alpha_{1} x_{1}^{n}+\alpha_{2} x_{2}^{n}+\alpha_{3} x_{3}^{n}, \\
B_{n}=\beta_{1} x_{1}^{n}+\beta_{2} x_{2}^{n}+\beta_{3} x_{3}^{n}, \\
C_{n}=\gamma_{1} x_{1}^{n}+\gamma_{2} x_{2}^{n}+\gamma_{3} x_{3}^{n},
\end{array}\right.
$$

where $x_{1}, x_{2}, x_{3}$ are the roots of (1), and where $\alpha_{i}, \beta_{i}, \gamma_{i}$ are determined by (5) using the initial values (3). These latter are readily found to be

$$
\left\{\begin{array}{cc}
\alpha_{1}=x_{1}^{2}\left(x_{2}-x_{3}\right) / D, & \alpha_{2}=x_{2}^{2}\left(x_{3}-x_{1}\right) / D, \\
\beta_{1}=x_{1}^{2}\left(x_{3}^{2}-x_{2}^{2}\right) / D, & \beta_{2}=x_{2}^{2}\left(x_{1}^{2}-x_{3}^{2}\right) / D, \\
\gamma_{1}=x_{1}^{2} x_{2} x_{3}\left(x_{2}-x_{3}\right) / D, & \gamma_{2}=x_{2}^{2} x_{1} x_{3}\left(x_{3}-x_{1}\right) / D, \\
\alpha_{3}=x_{3}^{2}\left(x_{1}-x_{2}\right) / D, \\
\beta_{3}=x_{3}^{2}\left(x_{2}^{2}-x_{1}^{2}\right) / D, \\
\gamma_{3}=x_{3}^{2} x_{1} x_{2}\left(x_{1}-x_{2}\right) D,
\end{array}\right.
$$

where

$$
D=\left(x_{2}-x_{1}\right)\left(x_{3}-x_{1}\right)\left(x_{3}-x_{2}\right) \text {. }
$$

In the quotient (4) substitute from (5) and simplify. The numerator becomes

$$
\begin{gathered}
\alpha_{1} \gamma_{2} x_{1}^{n} x_{2}^{n}\left(x_{2}-x_{1}\right)+\alpha_{1} \gamma_{3} x_{1}^{n} x_{3}^{n}\left(x_{3}-x_{1}\right)+\alpha_{2} \gamma_{1} x_{2}^{n} x_{1}^{n}\left(x_{1}-x_{2}\right) \\
+\alpha_{2} \gamma_{3} x_{2}^{n} x_{3}^{n}\left(x_{3}-x_{2}\right)+\alpha_{3} \gamma_{1} x_{3}^{n} x_{1}^{n}\left(x_{1}-x_{3}\right)+\alpha_{3} \gamma_{2} x_{3}^{n} x_{2}^{n}\left(x_{2}-x_{3}\right),
\end{gathered}
$$

and the denominator is the same expression with $\gamma_{i}$ replaced by $\beta_{i}$.

If now the roots of (1) are such that $\left|x_{1}\right|<\left|x_{2}\right|$ and also $\left|x_{1}\right|<\left|x_{3}\right|$ then

$$
\lim _{n \rightarrow \infty}\left|\frac{x_{1}}{x_{2}}\right|^{n}=0, \text { and } \lim _{n \rightarrow \infty}\left|\frac{x_{1}}{x_{3}}\right|^{n}=0 .
$$

Hence if the numerator and the denominator of (4) be divided by $x_{3}^{n} x_{3}^{n}$ the limit as $n$ becomes infinite is 


$$
-\frac{\left(\alpha_{2} \gamma_{3}-\alpha_{3} \gamma_{2}\right)}{\left(\alpha_{2} \beta_{3}-\alpha_{3} \beta_{2}\right)} ;
$$

but this reduces to $x_{1}$ on substituting from (6). Thus our method is justified.

The recursion formulas (2) may be thrown into a more useful form. From (5) and (6), we find

$$
\frac{C_{n+1}}{A_{n}}=x_{1} x_{2} x_{3}=c \text {. }
$$

In a similar way the second of the following formulas is proved :

$$
\left\{\begin{array}{l}
A_{n+1}=a A_{n}+b A_{n-1}+c A_{n-2}, \\
B_{n+1}=b A_{n}+c A_{n-1}, \\
C_{n+1}=c A_{n} .
\end{array}\right.
$$

These may be written in the form

$$
\left\{\begin{array}{l}
A_{n+1}=a A_{n}+B_{n}, \\
B_{n+1}=b A_{n}+C_{n}, \\
C_{n+1}=c A_{n} .
\end{array}\right.
$$

2. Application to Units in Pure Cubic Fields. In pure cubic fields defined by the real root of $x^{3}-R=0$, when $R$ contains no square factor and when the field is one of the first species, i.e., $R \neq \pm 1(\bmod 9),{ }^{*}$ a basis consists of the numbers 1 , $\sqrt[3]{R}, \sqrt[3]{R^{2}}$. All algebraic integers of the field are of the form

$$
X+Y \sqrt[3]{R}+Z \sqrt[3]{R^{2}}
$$

where $X, Y, Z$ are rational integers. The units of the field have their norms equal to \pm 1 . Hence in order to find the units we must solve the Diophantine cubic

$$
X^{3}+R Y^{3}+R^{2} Z^{3}-3 R X Y Z= \pm 1
$$

* Dedekind, Über die Anzahl der Idealklassen in reinen kubischen Körpern, Journal FÜR MAthematik, 1900, p. 40; Sommer, Vorlesungen über Zahlentheorie, p. 261. 
We shall obtain solutions of this equation when $R$ is of certain numerical forms and in special cases obtain the fundamental unit of the corresponding field.

The identity

$$
\left|\begin{array}{lll}
A_{n} & B_{n} & C_{n} \\
A_{n+1} & B_{n+1} & C_{n+1} \\
A_{n+2} & B_{n+2} & C_{n+2}
\end{array}\right|=c\left|\begin{array}{lll}
A_{n-1} & B_{n-1} & C_{n-1} \\
A_{n} & B_{n} & C_{n} \\
A_{n+1} & B_{n+1} & C_{n+1}
\end{array}\right|
$$

is easily proved by substituting from (2) the values of $A_{n+2}, B_{n+2}, C_{n+2}$ in the left member. Hence by induction and from the initial values (3) we find that

$$
\left|\begin{array}{lll}
A_{n} & B_{n} & C_{n} \\
A_{n+1} & B_{n+1} & C_{n+1} \\
A_{n+2} & B_{n+2} & C_{n+2}
\end{array}\right|=-c^{n+2} .
$$

By (7), this becomes

$$
\left|\begin{array}{lll}
A_{n} & A_{n-2} & A_{n-1} \\
A_{n+1} & A_{n-1} & A_{n} \\
A_{n+2} & A_{n} & A_{n+1}
\end{array}\right|=-c^{n} .
$$

Expanding and substituting

$$
\begin{aligned}
& A_{n+2}=a A_{n+1}+b A_{n}+c A_{n-1}, \\
& A_{n-2}=\frac{A_{n+1}-a A_{n}-b A_{n-1}}{c},
\end{aligned}
$$

we obtain

$$
\left\{\begin{array}{l}
A_{n+1}^{3}+(a b+c) A_{n}^{3}+c^{2} A_{n-1}^{3}-(3 c-a b) A_{n+1} A_{n} A_{n-1} \\
-2 a A_{n+1}^{2} A_{n}-b A_{n+1}^{2} A_{n-1}+\left(a^{2}-b\right) A_{n+1} A_{n}^{2} \\
+\left(a c+b^{2}\right) A_{n}^{2} A_{n-1}+2 b c A_{n} A_{n-1}^{2}+a c A_{n-1}^{2} A_{n+1}=c^{n+1}
\end{array}\right.
$$

In the equation $x^{3}=R$, set $x=y+\alpha$. The transformed equation is

$$
y^{3}=-3 \alpha y^{2}-3 \alpha^{2} y+R-\alpha^{3} .
$$

Using this as equation (1) for calculating the $A$ 's, $B$ 's, $C$ 's, we have

$$
a=-3 \alpha, \quad b=-3 \alpha^{2}, \quad c=R-\alpha^{3} .
$$


With these values of $a, b, c(10)$ may be written in the form

$$
\begin{aligned}
& {\left[A_{n+1}+2 \alpha A_{n}+\alpha^{2} A_{n-1}\right]^{3}+R\left[A_{n}+\alpha A_{n-1}\right]^{3}+R^{2} A_{n-1}^{3}} \\
& -3 R\left[A_{n+1}+2 \alpha A_{n}+\alpha^{2} A_{n-1}\right]\left[A_{n}+\alpha A_{n-1}\right] A_{n-1}=c^{n+1} .
\end{aligned}
$$

Hence the cubic (9) has the solution

$$
\left\{\begin{array}{l}
X=\left(A_{n+1}+2 \alpha A_{n}+\alpha^{2} A_{n-1}\right) / c^{(n+2) / 3}, \\
Y=\left(A_{n}+\alpha A_{n-1}\right) / c^{(n+1) / 3}, \\
Z=A_{n-1} / c^{(n+1) / 3} .
\end{array}\right.
$$

This solution is integral in the three following cases.

First, let $R$ be of the form $m^{3} l^{3} \pm m$. Taking $\alpha=m l$, we find from (8) and (3)

$$
A_{1}=-3 m l, \quad A_{2}=6 m^{2} l^{2}, \quad A_{3}=-9 m^{3} l^{3} \pm m ;
$$

therefore for $n=2$ we have from (11)

$$
X=1, \quad Y= \pm 3 m l^{2}, \quad Z=\mp 3 l .
$$

Second, let $R$ be of the form $l^{3} \pm 1$. Taking $\alpha=l$, we obtain, for $n=1$, the solution

$$
X= \pm l^{2}, \quad Y=\mp 2 l, \quad Z= \pm 1 \text {. }
$$

Third, let $R$ be of the form $l^{3} \pm 3$. With $\alpha=l$, we obtain, for $n=2$,

$$
X=1, \quad Y= \pm l^{2}, \quad Z=\mp l .
$$

If $R=l^{3}+1$, the unit given by (13), namely,

$$
\eta=l^{2}-2 l \sqrt[3]{R}+\sqrt[3]{R^{2}}
$$

is the square of the unit $\epsilon=\sqrt[3]{R}-l$. The unit $\epsilon$ for fields of the first species, ${ }^{*}$ that is $R \neq \pm 1(\bmod 9)$, and therefore $l \neq 0(\bmod 3)$, is the fundamental unit, all other units being expressible as powers of $\epsilon$. When the field is of the second species, and $l \equiv 0(\bmod 3)$, then $\epsilon$ is either the fundamental unit or the square of the fundamental unit and the latter alternative can occur in only a finite number of fields.

* Nagell, Solution complète de quelques equations d deux indeterminees, Journal de Mathematiques, 1925, p. 211. 
This follows immediately from a theorem of Delaunay as perfected by Nagell* since $\epsilon=\sqrt[3]{R}-l$ is of the form $x+\sqrt[3]{R} y$ where $x$ and $y$ are solutions of $x^{3}+R y^{3}=1$. In our case $x=-l, y=1, R=l^{3}+1$. Note that $0<\epsilon<1$. Corresponding results hold when $R=l^{3}-1$; then $\epsilon=l-\sqrt[3]{R}$.

When $R=m^{3} l^{3}+m$, the unit given by (12), namely, $\eta=1+3 m l^{2} \sqrt[3]{R}-3 l \sqrt[3]{R^{2}}=\frac{(\sqrt[3]{R}-m l)^{3}}{m}=\left(\sqrt[3]{m^{2} l^{3}+1}-l \sqrt[3]{m^{2}}\right)^{3}$, furnishes a solution of the equation

$$
m^{2} x^{3}+\left(m^{2} l^{3}+1\right) y^{3}=1,
$$

viz., $x=-l, y=1$. It is easily seen that $0<\eta<1$. Thus $\dagger$ $\eta=\epsilon^{k}$ where $k=2^{n}$ with $n=0,1,2, \cdots$, and $\epsilon$ is the fundamental unit in the field $k(\theta), \theta=\sqrt[3]{m^{2} b^{2} d}$, where $m^{2} l^{3}+1=b d^{2}$. When $m^{2} l^{3}+1$ contains no square as a factor, that is, when $d=1$, then $k(\theta)=k\left(\sqrt[3]{R^{2}}\right)$ is the same field as $k(\sqrt[3]{R})$.

Moreover if $m$ is even and $m^{2} l^{3}+1$ is divisible by a number of the form $8 n-1$ or $8 n+5$, then $\eta$ is the fundamental unit of the ring $\ddagger(1, \theta, \sqrt[3]{R})$. The ring reduces to the field $k(\sqrt[3]{R})$ when $m^{2} l^{3}+1$ contains no square factor and $\eta$ becomes the fundamental unit of the field. Corresponding results hold when $R=m^{3} l^{3}-m$; then

$$
\eta=1-3 m l^{2} \sqrt[3]{R}+3 l \sqrt[3]{R^{2}}=\frac{(m l-\sqrt[3]{R})^{3}}{m}
$$

The University of Nebraska

* Nagell, loc. cit., p. 234.

† Nagell, loc. cit., p. 249.

$\ddagger$ Nagell, loc. cit. pp. 252-258. 